\title{
Evidence of impurity and boundary effects on magnetic monopole dynamics in spin ice
}

\author{
H. M. Revell ${ }^{1,2}$, L. R. Yaraskavitch ${ }^{1,2}$, J. D. Mason ${ }^{1,2}$, K. A. Ross ${ }^{3}$, H. M. L. Noad ${ }^{3}$, H. A. Dabkowska ${ }^{4}$, \\ B. D. Gaulin ${ }^{3,4,5}$, P. Henelius ${ }^{6 \star}$ and J. B. Kycia ${ }^{1,2}$
}

Electrical resistance is a crucial and well-understood property of systems ranging from computer microchips to nerve impulse propagation in the human body. Here we study the motion of magnetic charges in spin ice and find that extra spins inserted in $\mathrm{Dy}_{2} \mathrm{Ti}_{2} \mathrm{O}_{7}$ trap magnetic monopole excitations and provide the first example of how defects in a spin-ice material obstruct the flow of monopoles-a magnetic version of residual resistance. We measure the time-dependent magnetic relaxation in $\mathrm{Dy}_{2} \mathrm{Ti}_{2} \mathrm{O}_{7}$ and show that it decays with a stretched exponential followed by a very slow long-time tail. In a Monte Carlo simulation governed by Metropolis dynamics we show that surface effects and a very low level of stuffed spins $(0.30 \%)$-magnetic Dy ions substituted for non-magnetic $\mathrm{Ti}$ ions-cause these signatures in the relaxation. In addition, we find evidence that the rapidly diverging experimental timescale is due to a temperature-dependent attempt rate proportional to the monopole density.

The exceptional physical properties of the spin-ice materials arise from the underlying pyrochlore lattice of corner-sharing tetrahedra and crystal-field effects, which constrain the magnetic moments of the rare-earth ions to point along the axis connecting the centres of the two neighbouring tetrahedra ${ }^{1}$. As a result, the spin-ice materials are highly frustrated and possess a ground state containing a large residual entropy similar to water ice ${ }^{2}$. The thermodynamic properties of the spin-ice materials have been very successfully modelled by the Hamiltonian for Ising spins interacting through dipolar and exchange interactions ${ }^{3,4}$,

$$
H=-J \sum_{\langle i j\rangle} \mathbf{S}_{\mathbf{i}} \cdot \mathbf{S}_{\mathbf{j}}+D r_{n n}^{3} \sum_{i>j} \frac{\mathbf{S}_{\mathbf{i}} \cdot \mathbf{S}_{\mathbf{j}}}{\left|\mathbf{r}_{i j}\right|^{3}}-\frac{3\left(\mathbf{S}_{\mathbf{i}} \cdot \mathbf{r}_{i j}\right)\left(\mathbf{S}_{\mathbf{j}} \cdot \mathbf{r}_{i j}\right)}{\left|\mathbf{r}_{i j}\right|^{5}}
$$

where $r_{n n}=3.5 \AA, D=1.41 \mathrm{~K}$ and $J=3.72 \mathrm{~K}$, and the moments $S$, of unit length, are forced to point along the local $\langle 111\rangle$ axes. Recently, it was realized that the fundamental excitations are magnetic charges, commonly referred to as monopoles ${ }^{5,6}$ that are created by overturning a spin in the highly degenerate spin-ice ground state, where two spins point in and two point out of each tetrahedron. The motion of magnetic monopoles has been observed experimentally through the generation of monopole currents by the application of a magnetic field ${ }^{7}$, and muon spin rotation ${ }^{8}$, which is a subject of recent controversy ${ }^{9}$. Monte Carlo simulations of a Coulomb gas of monopoles ${ }^{10}$ and the dipolar spin-ice model ${ }^{11}$, equation (1), agree well with experimental results down to $1 \mathrm{~K}$, below which the observed dynamics become much slower in the experiments than in the simulations ${ }^{11-17}$. In this study we find that significant corrections to the ideal model in equation (1) are necessary to accurately model the motion of magnetic monopoles in the real material. Similarly to electrical conductors and semiconductors, in which local impurities can decrease the conductivity, or introduce new states in the bandgap, we find that a small amount of extra spins and surface effects change the flow of magnetic monopoles.

Experimental access to the properties of the magnetic monopoles is provided through the dynamic correlation function $C(t)=\langle M(0) M(t)\rangle$, where $M(t)$ is the time-dependent magnetization of the sample. To study these excitations we therefore scrutinize the low-temperature dynamics of $\mathrm{Dy}_{2} \mathrm{Ti}_{2} \mathrm{O}_{7}$. In particular, we measure $C(t)$ by two independent methods using custom designed superconducting quantum interference device (SQUID) circuits. In the direct field-quench measurement we apply a small field of $5 \mathrm{mOe}$ to the sample and directly observe the decay of the magnetization. In the second method the imaginary part of the susceptibility, $\chi^{\prime \prime}(\omega)$, is recorded as a function of frequency, and transformed to the time domain using the fluctuation-dissipation theorem,

$$
C(t)=\langle M(0) M(t)\rangle=2 k T \int_{-\infty}^{\infty} \frac{\chi^{\prime \prime}(\omega)}{\omega} \cos (\omega t) \mathrm{d} \omega
$$

This transformation is very sensitive to the precise form of the susceptibility, and is much facilitated by an analytical expression for $\chi$ ". As in the case of $\mathrm{Ho}_{2} \mathrm{Ti}_{2} \mathrm{O}_{7}$ (ref. 15), the commonly used Debye, Davidson-Cole and Havriliak-Negami functions fail to capture the tails and asymmetry of the spectrum. Instead we fit the susceptibility to the functional form

$$
\chi^{\prime \prime}(\omega)=\frac{1}{\left[(\tau \omega)^{\alpha_{1} \cdot n}+(\tau \omega)^{-\alpha_{2} \cdot n}\right]^{\frac{1}{n}}}
$$

introduced to model the broad susceptibility spectrum of a spin glass $^{18}$. The position and sharpness of the maximum are given by $\tau$ and $n$, and $\chi^{\prime \prime}$ vanishes with exponents $\alpha_{1}$ and $\alpha_{2}$. Supplementary Fig. S1 demonstrates that this form is a substantial improvement on the single ${ }^{17}$ and double ${ }^{16}$ Debye models previously used.

The main result of this investigation is shown in Fig. 1, where we show the relaxation function $C(t)$ calculated using equation (2) together with the direct field-quench measurement, as well as the result of a dynamic Monte Carlo calculation. At low temperatures the standard dipolar spin-ice model predicts

\footnotetext{
${ }^{1}$ Department of Physics and Astronomy and Guelph-Waterloo Physics Institute, University of Waterloo, Waterloo, Ontario N2L 3G1, Canada, ${ }^{2}$ Institute for Quantum Computing, University of Waterloo, Waterloo, Ontario N2L 3G1, Canada, ${ }^{3}$ Department of Physics and Astronomy, McMaster University, Hamilton, Ontario L8S 4M1, Canada, ${ }^{4}$ Brockhouse Institute for Materials Research, McMaster University, Hamilton, Ontario L8S 4M1, Canada, ${ }^{5}$ Canadian Institute for Advanced Research, 180 Dundas Street West, Toronto, Ontario M5G 1Z8, Canada, ${ }^{6}$ Department of Theoretical Physics, Royal Institute of Technology, SE-106 91 Stockholm, Sweden. *e-mail: henelius@kth.se.
} 


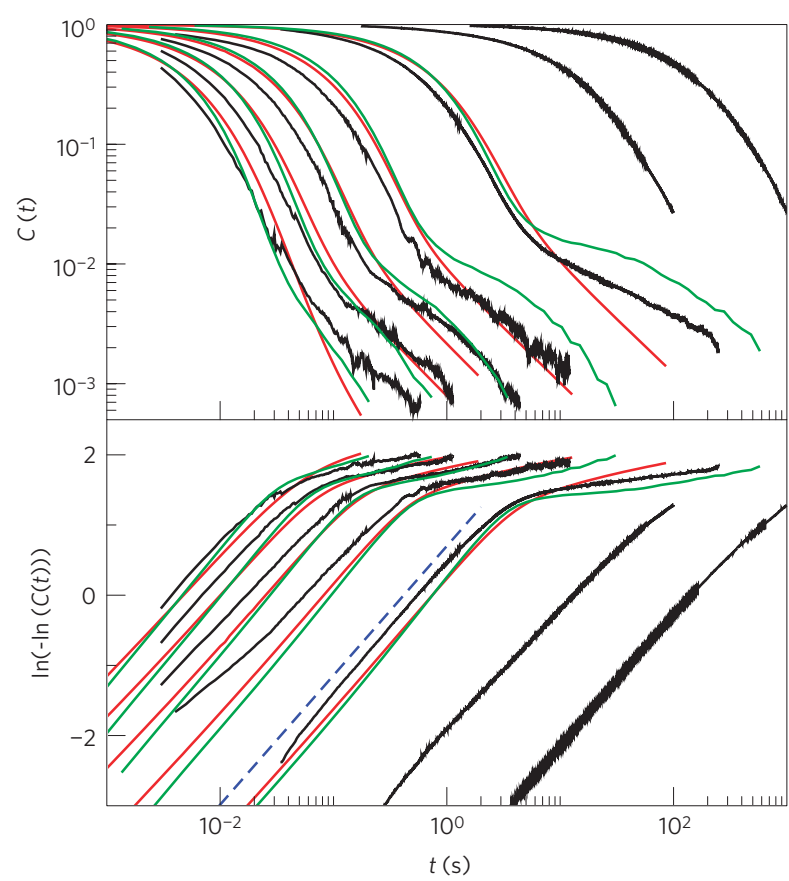

Figure 1 | Decay of magnetic correlations. $C(t)$ determined from equation (2) (red lines) along with a direct field-quench measurement (solid black line). Results from dynamic Monte Carlo simulations for $L=6$ with $0.30 \%$ stuffed spins $(3,456+10$ spins) are shown in green. The Monte Carlo time constant has been scaled to match experiments. Temperatures are 1.1, 1.0, $0.9,0.8,0.675,0.550$ and $0.475 \mathrm{~K}$ from left to right. The stretch exponent $\beta$ is given by the gradient of the lines in the lower panel. A dashed blue line with gradient $\beta=0.8$ is shown for comparison.

that the magnetic monopole excitations lead to an exponential decay of the magnetization ${ }^{10,11}, C(t, T)=\mathrm{e}^{-t / \tau(T)}$, where $\tau(T)$ is the temperature-dependent correlation time. The experimentally observed decay depicted in Fig. 1 differs in three important ways from this prediction. First, the decay is not a simple exponential function, but rather a stretched exponential function, $C(t)=\mathrm{e}^{(-t / \tau)^{\beta}}$. This is evident in the lower panel of Fig. 1, where we show $\ln (-\ln (C(t)))$ as a function of $\ln (t)$. A straight line fit to the data in the linear region yields a stretching exponent $\beta \approx 0.7-0.8$. Second, the stretched exponential decay is followed by a very slow long-time tail of the relaxation. Third, the Arrhenius scaling of the correlation time, $\tau(T)=\tau_{0} \exp E / T$, yields an energy barrier of about $9 \mathrm{~K}$, compared with about $5 \mathrm{~K}$ predicted by the dipolar spin-ice model.

These effects are surprising, because stretched exponential decay, algebraic tails and very slow dynamics are generally associated with disordered systems, such as spin-glass materials, whereas the spin-ice materials are expected to be relatively clean. Furthermore, the dipolar spin-ice model has been extremely successful in reproducing the static properties of the spin-ice materials, and these major discrepancies are unprecedented. We have therefore performed extensive dynamic Monte Carlo simulations to find the cause for this unexpected behaviour. To achieve the qualitative agreement with the experimental data shown in Fig. 1 we made three modifications to the standard dipolar spin-ice model. First, we used open boundary conditions instead of periodic boundary conditions, which caused the stretched exponential decay. Second, we included a small percentage $(0.30 \%)$ of extra (stuffed) spins that lead to the slow long-time tail. Finally, we allowed the conversion factor from Monte Carlo time to real time to be temperature dependent, a procedure we motivate below. The effects of these three additions are essentially independent and

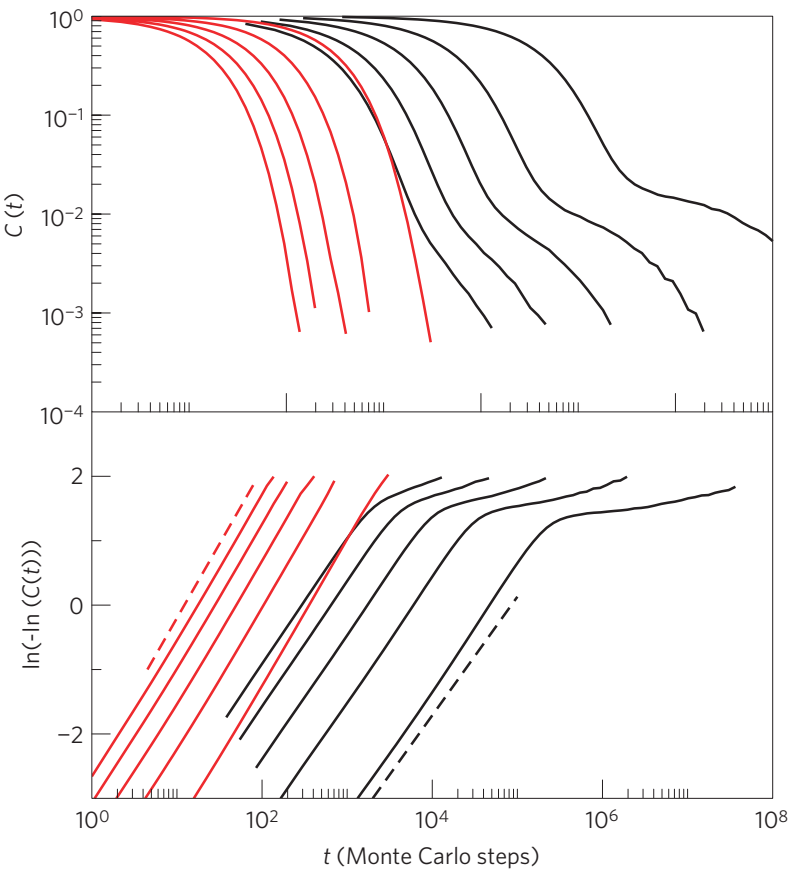

Figure 2 | Decay of correlations from simulations. Dynamic correlation function from Monte Carlo calculations using open boundary conditions (black lines) for $L=6$ with $0.30 \%$ stuffed spins $(3,456+10$ spins). The timescale has been adjusted to match the increasing experimental timescale. Red lines are results for the standard dipolar spin-ice model with periodic boundary conditions for $L=8$. The stretch exponent $\beta$ is given by the gradient of the lines in the lower panel. Dashed red and blue lines with gradients $\beta=1.0$ and $\beta=0.8$ are shown for comparison. Temperatures are $1.1,1.0,0.9,0.8$ and $0.675 \mathrm{~K}$ from left to right.

we find that all three modifications are needed to obtain a good description of the experimental data. In Fig. 2 we explicitly compare the correlation function for the dipolar spin-ice model with and without modifications.

Conventional practice regarding simulations of dipolar-coupled magnets is to use periodic boundary conditions and Ewald summation ${ }^{10}$. However, owing to effects such as charge build-up ${ }^{10}$ one may expect corrections from an open boundary. Therefore, we use open boundary conditions to investigate the effect a surface may have on magnetic relaxation and monopole dynamics. We find that the decay changes from exponential to stretched exponential, and the stretched decay is quite stable as the system size is increased from $L=4$ (1,024 spins) to $L=10$ (16,000 spins). Static quantities such as the specific heat also appear to converge to slightly different limits depending on whether one uses open or periodic boundary conditions. It is interesting to note that unexpected surface behaviour arising from proton disorder in water ice has recently been discovered ${ }^{19,20}$. Next, we consider the effect of stuffed spins on the long-time decay in more detail.

The non-magnetic Ti ions in $\mathrm{Dy}_{2} \mathrm{Ti}_{2} \mathrm{O}_{7}$ sit on a pyrochlore lattice shifted from the pyrochlore lattice of the Dy ions. It is possible to substitute Ti sites with Dy ions, thereby increasing the connectivity of the Dy pyrochlore lattice. Previous work has considered higher levels $(>10 \%)$ of stuffing ${ }^{21,22}$, but here we investigate the dynamic effects of a very low level $(<1 \%)$ of stuffing, which we suspect may occur in our samples. This scenario is supported by a recent crystallographic study showing stuffing at the per cent level in nominally clean samples of $\mathrm{Yb}_{2} \mathrm{Ti}_{2} \mathrm{O}_{7}$ (ref. 23).

In this study we made the simplest assumption that the stuffed spins are Ising spins ${ }^{21}$ of magnitude $10 \mu_{\mathrm{B}}$ forced to point along the axis between centres of the Ti tetrahedra. The stuffed spin has 


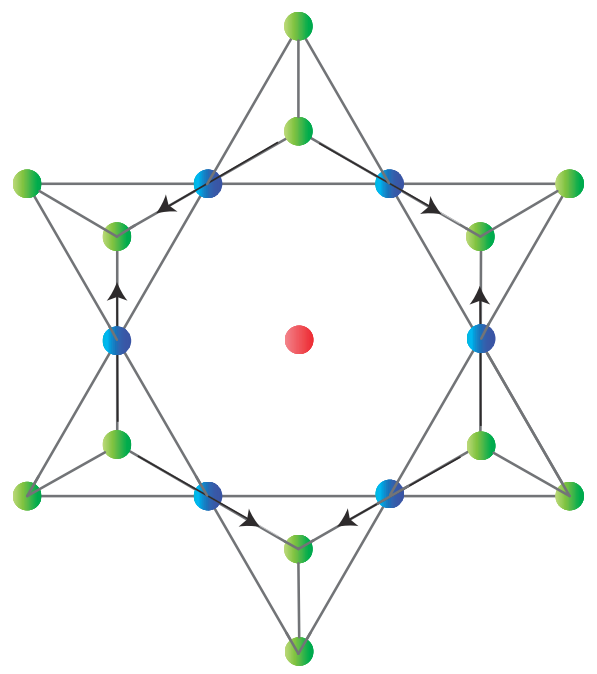

Figure 3 | Tetrahedra surrounding a stuffed spin. The crystal environment surrounding a stuffed spin, which is indicated by the red central dot. The blue dots are nearest neighbours to the stuffed spin, with arrows indicating the local Ising axis. The remaining corners of the nearest tetrahedra are shown in green.

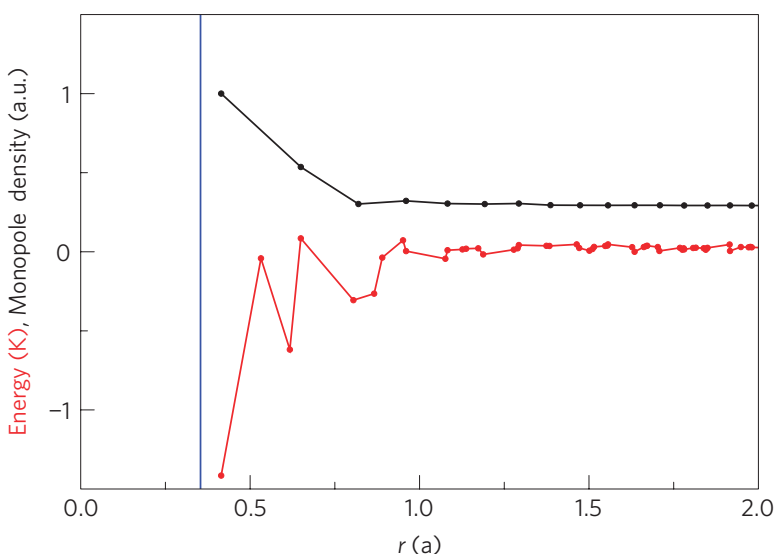

Figure 4 | Density and energy of monopoles surrounding a stuffed spin. Monte Carlo density (black curve) of monopoles as a function of distance from a single stuffed spin ( $r$ ) at $T=0.675 \mathrm{~K}$. The red curve shows the energy of spin-ice configurations containing one monopole pair as a function of pair distance from the stuffed spin. The energy is recorded only for configurations in which the monopoles occupy adjacent tetrahedra. The vertical blue line indicates the nearest-neighbour spin distance.

six nearest-neighbour spins and interacts with the same nearestneighbour exchange interaction as the ordinary spins. The six tetrahedra surrounding the stuffed spin are depicted in Fig. 3. The average energy barrier to flipping a nearest-neighbour spin increases from $5.8 \mathrm{~K}$ (for ordinary Dy spins) to $8.6 \mathrm{~K}$, whereas it is $14.5 \mathrm{~K}$ for the stuffed spin. Despite these high barriers we find that the monopole density in the vicinity of the stuffed spin is higher than in the rest of the material, and the energy is lower, as depicted in Fig. 4. The impurity, in effect, traps the monopoles, and Supplementary Movie S1 generated by the Monte Carlo simulation visualizes how a monopole is attracted to a stuffed spin.

In addition to attracting existing monopoles, we find that a stuffed spin also causes an increase in the number of monopoles created next to it. The high-energy barrier of $8.6 \mathrm{~K}$ for flipping a nearest-neighbour spin holds only in the lowest energy configuration shown in Fig. 3, where the two nearest-neighbour spins belonging to each tetrahedron both point either in or out of the

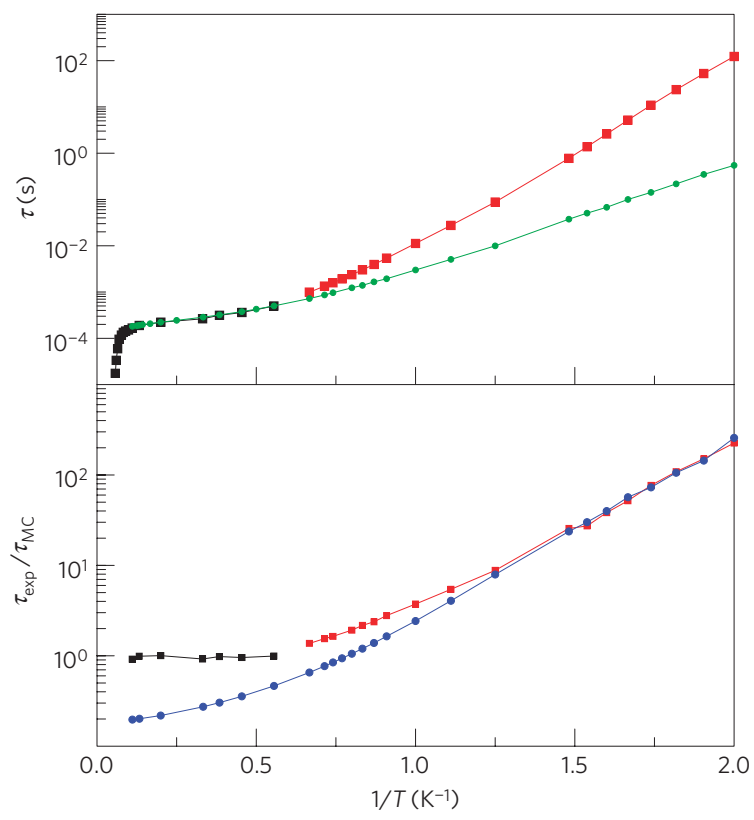

Figure 5 | Temperature dependence of the correlation time $\tau$. Experimentally determined correlation time (red squares) along with experimental results from ref. 16 (black squares) and Monte Carlo results in green. One Monte Carlo step corresponds to $0.26 \mathrm{~ms}$, matching Monte Carlo and experimental data at $5 \mathrm{~K}$. In the lower panel the ratio of the experimental data to Monte Carlo results is shown in red and black squares along with the inverse Monte Carlo monopole density (blue circles), matched to experimental data in the low-temperature limit.

tetrahedra. If a monopole passes by the stuffed spin and overturns one of the nearest-neighbour spins, but still leaves the surrounding tetrahedra in a 2-in 2-out spin-ice ground state, then the barrier to overturning a nearest-neighbour spin decreases to $2 \mathrm{~K}$, causing a large increase in the number of monopoles created near the stuffed spin. This effect can be viewed in Supplementary Movie S2.

Finally we address the rapidly increasing correlation time observed when lowering the temperature. As is evident from Fig. 5, the correlation time calculated from the Monte Carlo simulation agrees well with experiments above $1.5 \mathrm{~K}$, but falls rapidly below the experimental values at lower temperatures. In this limit the correlation time is expected to depend on the monopole density $\rho$ and the attempt rate $1 / \tau_{0}$ as $\tau \propto \tau_{0} / \rho$ (ref. 24). Therefore, the experimental to Monte Carlo correlation-time ratio $\tau^{\exp } / \tau^{\mathrm{MC}}=\tau_{0}^{\exp } / \tau_{0}^{\mathrm{MC}}$ is expected to be a constant, which is the case above $1.5 \mathrm{~K}$, as can be seen from the lower panel in Fig. 5. Below $1.5 \mathrm{~K}$ this ratio is not a constant, and below $0.7 \mathrm{~K}$ the ratio grows like the inverse monopole density, $\rho^{-1} \propto \exp (E / T)$, with $E=4.5 \mathrm{~K}$. However, theory and experiments can be reconciled if we assume a temperature-dependent attempt rate in the material and, in particular, a low-temperature dependence of the form $\tau_{0}^{\exp } \propto 1 / \rho$. Physically this would mean that the strong and fluctuating local fields caused by the motion of the monopoles directly affect the spin-flip attempt rate. In this work we have assumed that the attempt rate in the real material is temperature dependent below $1.5 \mathrm{~K}$ and rescaled the Monte Carlo time accordingly. Furthermore, this implies that the correlation time scales with monopole density as $\tau^{\exp } \propto 1 / \rho^{\eta_{\exp }}$, with $\eta_{\exp }=2$ and $\eta_{\exp }=1$ in the low- and high- temperature limits (until the low-density assumption fails). This extrapolates well with the phenomenological values of $\eta_{\exp }=2 / 3$ and $\eta_{\exp }=3 / 4$ found at intermediate temperatures ${ }^{24}$. Finally, note that the plateau value of $\tau$ in the upper panel of Fig. 5 is about $0.1 \mathrm{~ms}$ rather than the generally assumed value of $1 \mathrm{~ms}$. This is due to a factor $2 \pi$ necessary in the transformation of experimental frequency to time, $\tau=1 /(2 \pi f)$. 
Our work demonstrates the necessity of taking subtle material defects and surface effects into account to properly describe the magnetic relaxation and the motion of magnetic charges in the spin-ice materials. This leads to interesting new aspects for the physics of monopoles in spin ice. Experimentally, the structural characteristics of the material should be carefully investigated. Modifying or reducing material defects and the surface structure could alter the transport properties in fundamental ways, similarly to metals. Theoretically, considering altered easy-axis and exchange couplings at stuffed spins, stacking faults as well as surfaces may improve quantitative agreement with experiments. We hope that the present study stimulates further investigations into the effects of disorder on magnetic charge transport.

\section{Methods}

The single-crystal $\mathrm{Dy}_{2} \mathrm{Ti}_{2} \mathrm{O}_{7}$ samples were prepared at McMaster University, using a floating-zone image furnace technique ${ }^{25}$ with a growth rate of $6 \mathrm{~mm} \mathrm{~h}^{-1}$ in $3 \mathrm{~atm}$ of $\mathrm{O}_{2}$. The samples were studied by performing a.c. susceptibility ${ }^{17}$ and direct field-quench measurements at the University of Waterloo. Field-quench measurements of the bulk magnetic moment were obtained using a SQUID magnetometer mounted on a dilution refrigerator. The use of a SQUID ensured no loss of sensitivity at low frequency. Magnetic shielding was used to isolate the sample from the Earth's field. The geometry of the sample used in the field-quench measurements was a needle of dimension $0.5 \times 0.5 \times 4.3 \mathrm{~mm}^{3}$. A d.c. magnetic field of $5 \mathrm{mOe}$ was applied to the sample along the [100] crystal axis $\left( \pm 2^{\circ}\right)$, using a superconducting coil. Measuring the magnetization of the sample along the length of the needle minimized the demagnetizing effects. It was verified that the magnitude of the field was small enough so that the magnetization remained in the linear regime. In performing the field-quench measurements, the field was applied until the magnetization of the sample had equilibrated. The field was then turned off and the decay of the magnetization was observed.

The cubic Monte Carlo cell contained $L^{3}$ unit cells and $16 \times L^{3}$ sites. On average, one attempt to flip each spin was made during each Monte Carlo step. We used $10^{6}$ equilibration Monte Carlo steps, followed by $10^{7}$ Monte Carlo steps during which measurements were taken. Stuffing was implemented by randomly occupying $0.3 \%$ sites of the Ti lattice and averaging over 100 disorder configurations.

Received 2 August 2012; accepted 28 September 2012; published online 11 November 2012

\section{References}

1. Harris, M. J., Bramwell, S. T., McMorrow, D. F., Zeiske, T. \& Godfrey, K. W. Geometrical frustration in the ferromagnetic pyrochlore $\mathrm{Ho}_{2} \mathrm{Ti}_{2} \mathrm{O}_{7}$. Phys. Rev. Lett. 79, 2554-2557 (1997).

2. Ramirez, A., Hayashi, A., Cava, R., Siddharthan, R. \& Shastry, B. Zero-point entropy in 'spin-ice'. Nature 399, 333-336 (1999).

3. Siddharthan, R. et al. Ising pyrochlore magnets: Low-temperature properties, ice rules and beyond. Phys. Rev. Lett. 83, 1854-1857 (1999).

4. Den Hertog, B. C. \& Gingras, M. J. P. Dipolar interactions and origin of spin ice in Ising pyrochlore magnets. Phys. Rev. Lett. 84, 3430-3433 (2000).

5. Ryzhkin, I. A. Magnetic relaxation in rare-earth oxide pyrochlores. J. Exp. Theoret. Phys. 101, 481-486 (2005).

6. Castelnovo, C., Moessner, R. \& Sondhi, S. L. Magnetic monopoles in spin ice. Nature 451, 42-45 (2008).

7. Giblin, S. R., Bramwell, S. T., Holdsworth, P. C. W., Prabhakaran, D. \& Terry, I. Creation and measurement of long-lived magnetic monopole currents in spin ice. Nature Phys. 7, 252-258 (2011).
8. Bramwell, S. T. et al. Measurement of the charge and current of magnetic monopoles in spin ice. Nature 461, 956-960 (2009).

9. Dunsiger, S. R. et al. Spin ice: Magnetic excitations without monopole signatures using muon spin rotation. Phys. Rev. Lett. 107, 207207 (2011).

10. Jaubert, L. \& Holdsworth, P. Signature of magnetic monopoles and Dirac string dynamics in spin ice. Nature Phys. 5, 258-261 (2009).

11. Jaubert, L. D. C. \& Holdsworth, P. C. W. Magnetic monopole dynamics in spin ice. J. Phys. Condens. Matter 23, 164222 (2011).

12. Matsuhira, K., Hinatsu, Y. \& Sakakibara, T. Novel dynamic magnetic properties in the spin ice compound $\mathrm{Dy}_{2} \mathrm{Ti}_{2} \mathrm{O}_{7}$. J. Phys. Condens. Matter 13, L737-L746 (2001).

13. Snyder, J., Slusky, J. S., Cava, R. J. \& Schiffer, P. How 'spin-ice' freezes. Nature 413, 48-51 (2001).

14. Snyder, J. et al. Low-temperature spin freezing in the $\mathrm{Dy}_{2} \mathrm{Ti}_{2} \mathrm{O}_{7}$ spin ice. Phys. Rev. B 69, 064414 (2004).

15. Quilliam, J. A., Yaraskavitch, L. R., Dabkowska, H. A., Gaulin, B. D. \& Kycia, J. B. Dynamics of the magnetic susceptibility deep in the coulomb phase of the dipolar spin ice material $\mathrm{Ho}_{2} \mathrm{Ti}_{2} \mathrm{O}_{7}$. Phys. Rev. B 83, 094424 (2011).

16. Matsuhira, K. et al. Spin dynamics at very low temperature in $\mathrm{Dy}_{2} \mathrm{Ti}_{2} \mathrm{O}_{7}$. J. Phys. Soc. Jpn 80, 123711 (2011).

17. Yaraskavitch, L. R. et al. Spin dynamics in the frozen state of the dipolar spin ice material $\mathrm{Dy}_{2} \mathrm{Ti}_{2} \mathrm{O}_{7}$. Phys. Rev. B 85, 020410 (2012).

18. Biltmo, A. \& Henelius, P. Unreachable glass transition in dilute dipolar magnet. Nature Commun. 3, 857 (2012).

19. Ryzhkin, I. A. \& Petrenko, V. F. Violation of ice rules near the surface: A theory for the quasiliquid layer. Phys. Rev. B 65, 012205 (2001).

20. Watkins, M. et al. Large variation of vacancy formation energies in the surface of crystalline ice. Nature Mater. 10, 794-798 (2011).

21. Lau, G. C. et al. Zero-point entropy in stuffed spin-ice. Nature Phys. 2, 249-253 (2006).

22. Ehlers, G. et al. Dynamic spin correlations in stuffed spin ice $\mathrm{Ho}_{2+x} \mathrm{Ti}_{2-x} \mathrm{O}_{7-\delta}$. Phys. Rev. B 77, 052404 (2008).

23. Ross, K. A. et al. Single crystals of $\mathrm{Yb} 2 \mathrm{Ti} 2 \mathrm{O} 7$ grown by the Optical Floating Zone technique: naturally 'stuffed' pyrochlores? Phys. Rev. B preprint at http://arxiv.org/abs/1208.2281 (2012).

24. Castelnovo, C., Moessner, R. \& Sondhi, S. L. Debye-Hückel theory for spin ice at low temperature. Phys. Rev. B 84, 144435 (2011).

25. Gardner, J. S., Gaulin, B. D. \& Paul, D. M. Single crystal growth by the floating-zone method of a geometrically frustrated pyrochlore antiferromagnet, $\mathrm{Tb}_{2} \mathrm{Ti}_{2} \mathrm{O}_{7}$. J. Cryst. Growth 191, 740-745 (1998).

\section{Acknowledgements}

We thank M. Gingras, C. Henley and J. Lidmar for discussions. P.H. is grateful for the hospitality of Åbo Akademi, the computer support of PDC (Ferlin) at KTH and the financial support by the Stenbäck foundation. We acknowledge support from NSERC and CFI.

\section{Author contributions}

K.A.R., H.M.N., H.A.D. and B.D.G. prepared the crystals and H.M.R., L.R.Y., J.D.M and J.B.K. designed and performed magnetic measurements. P.H. performed the Monte Carlo simulations. The manuscript was written by P.H., H.M.R., L.R.Y. and J.B.K. with input and discussion from all authors.

\section{Additional information}

Supplementary information is available in the online version of the paper. Reprints and permissions information is available online at www.nature.com/reprints. Correspondence and requests for materials should be addressed to P.H.

\section{Competing financial interests}

The authors declare no competing financial interests. 